\title{
Produção e qualidade de melancia cultivada com água de diferentes salinidades e doses de nitrogênio
}

\author{
Andréa R. F. C. da Costa ${ }^{1}$, José F. de Medeiros ${ }^{2}$, Francisco de Q. Porto Filho ${ }^{2}$, \\ José S. da Silva ${ }^{3}$, Fabricia G. B. Costa ${ }^{4} \&$ Djanira C. de Freitas ${ }^{3}$
}

\begin{abstract}
RESUMO
O uso de águas salinas na agricultura é um dos principais fatores que limitam a produção e a qualidade dos frutos no Estado do Rio Grande do Norte, sendo a seleção de híbridos tolerantes à salinidade e a utilização de adubação nitrogenada, alternativas para se evitar perdas de rendimento e qualidade desses frutos. Assim, o objetivo com este trabalho foi avaliar o comportamento produtivo e a qualidade dos frutos de três cultivares de melancia (Quetzali, Shadow e Leopard) submetidas a diferentes salinidades da água de irrigação e doses de nitrogênio, na região de Mossoró, RN. Os tratamentos consistiram em aplicação de água de irrigação com cinco condutividades elétricas $\left(0,57 ; 1,36 ; 2,77 ; 3,86\right.$ e $\left.4,91 \mathrm{dS} \mathrm{m}^{-1}\right)$ e três doses de nitrogênio (48, 96 e $\left.144 \mathrm{~kg} \mathrm{ha}^{-1}\right)$, delineados em blocos casualizados, com quatro repetições. Foram avaliados a produtividade, o número e o peso médio dos frutos e o teor de sólidos solúveis. O peso médio e o número de frutos influenciaram as perdas de rendimento das cultivares de melancia estudadas; apenas a cultivar Shadow apresentou o teor de sólidos solúveis dos frutos alterado com a salinidade da água de irrigação.
\end{abstract}

Palavras-chave: Citrullus lanatus L., cultivares, condutividade elétrica, adubação nitrogenada

\section{Production and quality of watermelon cultivated with water of different salinities and doses of nitrogen}

\begin{abstract}
The use of saline waters in the agriculture is one of the main factors that lim it the production and the fruit quality in the State of Rio Grande do Norte, being the selection of tolerant hybrids to salinity and the use of the nitrogen fertilization, alternatives to avoid losses in income and quality of the fruits. Therefore, the objective of this study was to evaluate the production and the quality of the fruits of three cultivars of watermelon (Quetzali, Shadow and Leopard) under different salinities of irrigation water and doses of nitrogen in the region of Mossoró, RN. The treatments consisted in the application of irrigation water with five electrical conductivities $\left(0.57 ; 1.36 ; 2.77 ; 3.86\right.$ e $\left.4.91 \mathrm{dS} \mathrm{m}^{-1}\right)$ and three doses of nitrogen $(48,96 \mathrm{e}$ $144 \mathrm{~kg} \mathrm{ha}^{-1}$ ), delineated in randomized blocks, with four replications. The productivity, the number and the mean weight of fruits, as well as the content of soluble solids were evaluated. The mean weight of fruits and the number of fruits affected the loss of income of studied watermelons cultivars. Only the Shadow cultivar had the soluble solids content of fruits altered with the salinity of irrigation water.
\end{abstract}

Key words: Citrullus lanatus L., cultivars, electrical conductivity, nitrogen fertilization

\footnotetext{
${ }^{1}$ Doutoranda em Engenharia Agrícola/UFRPE. Rua Dom Manoel de Medeiros, s/n, Dois Irmãos, CEP 52171-900, Recife, PE. Fone: (81) 3320-6220. E-mail: andrearaquel19@hotmail.com

${ }^{2}$ DCAT/UFERSA. Av. Francisco Mota, 572, Costa e Silva, CEP 59625-900, Mossoró, RN. Fone: (84) 3315-1575. E-mail: jfmedeir@hotmail.com; porto@ufersa.edu.br

Engenheiro(a) Agrônomo(a),UFERSA. Email: silereudo.silva@ifrn.edu.br; djanira_freitas@hotmail.com

${ }^{4}$ Mestre em Irrigação e Drenagem, UFERSA. Email: fabricia_gratyelli@hotmail.com
} 


\section{INTRODUÇÃO}

A melancia é uma das principais espécies olerícolas cultivadas no Brasil, em especial na região Nordeste, por apresentar condições de solo e clima favoráveis ao seu cultivo podendo ser cultivada o ano inteiro sob condições irrigadas.

No estado do Rio Grande do Norte, mais precisamente no polo agrícola Assu-Mossoró, a melancia deixou de ser uma cultura explorada apenas no período das chuvas, quando sua produção era destinada a mercados locais, para se tornar uma atividade tecnificada, praticada por pequenos e médios produtores como, também, por empresas, destinando-se a produção a grandes mercados como o CEAGESP/SP e, mais recentemente, ao mercado externo (Torres, 2007).

De importante valor nutritivo, o consumo de melancia não é maior devido ao grande tamanho dos frutos e à dificuldade no descascamento, tornando seu preparo muito trabalhoso (Miguel et al., 2007). Por isto a tendência, tanto no Brasil quanto em outros países é, nos últimos anos, a preferência por frutos pequenos, o que permite o consumo total e imediato contribuindo para a redução das perdas de alimentos. Além do tamanho outros aspectos são igualmente importantes e devem ser relacionados ao desenvolvimento do fruto para realização da colheita, como polpa vermelha, o teor de sólidos solúveis acima de $8 \%$, o formato arredondado do fruto e a resistência a doenças (Ferreira et al., 2003).

Atualmente, a melancia sem sementes é também um produto muito aceito nos principais mercados do mundo e tem surgido como alternativa de cultivo para os produtores de hortaliças. No Brasil, sua produção ainda é incipiente tendo em vista o alto custo das sementes. Entretanto, na região de Mossoró a área cultivada nos últimos anos tem crescido substancialmente passando de 2000 ha (Costa et al., 2010).

A região apresenta não apenas condições favoráveis ao cultivo mas também disponibilidade de água. A vantagem desta água reside no grande potencial volumétrico ainda não utilizado e por sua exploração ser mais econômica em relação às águas de boa qualidade (Medeiros et al., 2003) porém a mesma é proveniente de poço que explora o aquífero Jandaíra, que tem o inconveniente de apresentar elevados teores de sais. Os efeitos dos sais sobre as plantas são de natureza osmótica, tóxica ou nutricional (Viana et al., 2004) e podem ser notados pelas dificuldades de absorção de água salina, pela interferência dos sais nos processos fisiológicos ou mesmo por toxidez, similares àquelas de adubações excessivas (Queiroga et al., 2006). Assim, a utilização dessas águas fica condicionada à tolerância da espécie da cultivar e ao manejo da irrigação com vistas ao controle de salinização dessas áreas.

$\mathrm{O}$ uso de $\mathrm{N}$ é uma alternativa para se reduzir o efeito da salinidade sobre as culturas (Kafkafi, 1984) enquanto a cultura da melancia, a exemplo de outras olerícolas tem, na nutrição mineral, um dos fatores que contribuem diretamente na produtividade e na qualidade dos frutos (Andrade Júnior et al., 2005). Desta maneira, para se alcançar uma boa produtividade e frutos de boa qualidade, o manejo da água de irrigação, tal como da adubação de olerícolas como a melancia, deve ser feito de maneira criteriosa durante o ciclo da cultura, não permitindo desperdício pelos produtores.
Carmo (2009) observou, avaliando o efeito de diferentes doses de $\mathrm{N}\left(\mathrm{N} 1=55, \mathrm{~N} 2=106\right.$ e N3 $\left.=156 \mathrm{~kg} \mathrm{ha}^{-1}\right)$ sobre a cultura da melancia, resposta significativa com o aumento nas doses de $\mathrm{N}$ sobre a produtividade da melancia. A maior produtividade foi obtida para a maior dose de $\mathrm{N}$ aplicada. Foi observada também diferença significativa no número de frutos por planta em resposta ao aumento na dose de $\mathrm{N}$.

Figueirêdo (2008) verificou, estudando o efeito de níveis de salinidade da água de irrigação na produção da melancia, que não houve efeito significativo para a produção comercial (PRC) e total (PRT); houve apenas tendência de decréscimo linear com incremento da salinidade da água de irrigação.

Mousinho et al. (2003) verificaram, em experimento no município de Fortaleza, CE, que a aplicação de diferentes doses de nitrogênio $\left(0,75,150\right.$ e $\left.300 \mathrm{~kg} \mathrm{ha}^{-1}\right)$ influenciou os sólidos solúveis em frutos de melancia, reduzindo-os com o aumento da dose.

Assim, o objetivo com este trabalho foi avaliar o comportamento produtivo e qualitativo de três cultivares de melancia submetidas a diferentes salinidades da água de irrigação e doses de nitrogênio aplicadas via fertirrigação, na região de Mossoró, no Estado do Rio Grande do Norte.

\section{Material e Métodos}

O experimento foi realizado no período de agosto a novembro de 2009, na Fazenda Experimental Rafael Fernandes, localizada em Alagoinha, distando $20 \mathrm{~km}$ da cidade de Mossoró, RN. O campo experimental pertence à Universidade Federal Rural do Semiárido - UFERSA, cujas coordenadas de referência são: $5^{\circ} 0$ ' $37^{\prime \prime} \mathrm{S}, 37^{\circ} 23^{\prime} 50^{\prime}$ 'W Gr e $72 \mathrm{~m}$ altitude.

O solo da área experimental foi classificado, conforme metodologia proposta pela EMBRAPA (1999) como Argissolo Vermelho-Amarelo.

A água de irrigação disponível na Fazenda Experimental foi proveniente de um poço perfurado do aquífero Arenito Açu que apresentava profundidade de aproximadamente $1000 \mathrm{~m}$, com condutividade elétrica da água (CEa) em torno de $0,57 \mathrm{dS} \mathrm{m}^{-1}$.

Os tratamentos consistiram em aplicação de água de irrigação com cinco níveis de condutividades elétricas (CE1 $=0,57, \mathrm{CE} 2=1,36, \mathrm{CE} 3=2,77, \mathrm{CE} 4=3,86$ e $\mathrm{CE} 5=4,91$ $\left.\mathrm{dS} \mathrm{m}^{-1}\right)$ e três doses de $\mathrm{N}$ aplicadas via fertirrigação $(48,96$ e $\left.144 \mathrm{~kg} \mathrm{ha}^{-1}\right)$ sendo a dose padrão de $96 \mathrm{~kg} \mathrm{ha}^{-1}(100 \%)$ e as doses $48 \mathrm{~kg} \mathrm{ha}^{-1}(50 \%)$ e $144 \mathrm{~kg} \mathrm{ha}^{-1}(150 \%)$, arranjados no esquema de parcela subdividida $5 \times 3$ e delineados em blocos completos casualizados com quatro repetições. Desta forma, a água de menor condutividade elétrica (CE1) foi proveniente do poço artesiano profundo e a água de maior condutividade elétrica (CE5) produzida previamente com a adição de sais $\left(\mathrm{NaCl}, \mathrm{CaCl}_{2} \cdot 2 \mathrm{H}_{2} \mathrm{O}\right.$ e $\mathrm{MgSO}_{4} \cdot 7 \mathrm{H}_{2} \mathrm{O}$, de modo que a proporção de $\mathrm{Na}, \mathrm{Ca}$ e $\mathrm{Mg}$, fosse 7:2:1 em $\mathrm{mmol}_{\mathrm{c}} \mathrm{L}^{-1}$ ); as outras três condutividades elétricas da água foram obtidas da mistura dessas duas águas, sendo monitoradas diariamente.

Já as três doses de nitrogênio foram produzidas com o arranjo das linhas de gotejadores da seguinte forma: em todo o experimento foram instaladas duas linhas de gotejadores por fileira de planta, em que numa dessas duas linhas foi fornecida 
a adubação nitrogenada enquanto a outra complementava a lâmina de irrigação e os demais nutrientes. Como a concentração do nutriente na água no interior do sistema era constante, as linhas laterais tinham suas vazões por metro de tubulação variável $\left(2,5 \mathrm{~L} \mathrm{~h}^{-1} \mathrm{~m}^{-1}\right.$ - nível $\mathrm{N} 1,5,0 \mathrm{~L} \mathrm{~h}^{-1} \mathrm{~m}^{-1}$ - nível $\mathrm{N} 2$ e $7,5 \mathrm{~L} \mathrm{~h}^{-1} \mathrm{~m}^{-1}$-nível N3) de modo que num dado tempo de irrigação a quantidade de água, de $\mathrm{N}$ e dos demais nutrientes aplicados era proporcional à vazão.

Para compensar o volume de água aplicada a outra linha paralela aplicava o mesmo tempo de irrigação e tinha vazão complementar, ou seja, 7,5, 5,0 e 2,5 $\mathrm{L} \mathrm{h}^{-1} \mathrm{~m}^{-1}$, respectivamente, para as subparcelas que recebiam as doses N1, N2 e N3.

O espaçamento utilizado no experimento foi o de $2,0 \times 0,5$ $\mathrm{m}$ entre plantas. As parcelas experimentais foram constituídas de três fileiras de plantas de $20 \mathrm{~m}$, sendo duas com a cultivar Shadow (sem sementes) e a outra com a cultivar Leopard (sem sementes). Para Shadow, as plantas de uma fileira foram utilizadas para análise de crescimento e a outra para avaliar o rendimento.

$\mathrm{O}$ arranjo das plantas foi feito de modo que ficasse uma planta de fruto com sementes, no caso a cultivar Quetzali, alternada com uma planta de fruto sem sementes, cultivares Shadow e Leopard, em cada linha da parcela experimental.

O sistema de irrigação utilizado no experimento foi por gotejamento. $\mathrm{O}$ manejo da irrigação foi realizado com base na estimativa da evapotranspiração máxima da cultura (ETm) diariamente, conforme método proposto pela FAO 56 (Allen et al., 2006), aplicando-se a metodologia do Kc dual. Os valores de Kcb (Kc basal da cultura) recomendados pela FAO 56 são iguais a 0,$15 ; 0,95$ e 0,70 para melancia, nas fases inicial e intermediária e no final, respectivamente.

Três colheitas foram realizadas nos dias 10,18 e 20 de novembro de 2009 utilizando-se as cultivares Quetzali, Shadow e Leopard para análise dos componentes de produção.

A área útil de cada cultivar por parcela foi de 10 $\mathrm{m}^{2}$ correspondendo a 5 plantas da fileira. Os frutos de cada subparcela foram pesados e classificados em frutos comerciáveis ou refugo (frutos pequenos, manchados, atacados por pragas e doenças). A produção total foi a soma dos frutos comerciais com os refugos.

Os seguintes componentes de produção foram avaliados: peso médio dos frutos comerciais (PMC) e peso médio dos frutos totais (PMT) expressos em kg; número médio de frutos comerciais (NFC) e número médio de frutos totais (NFT). Para a produtividade foram tomados os dados de produtividade comercial (PRODC) e produtividade total (PRODT) expressos em Mg ha-1.

Por ocasião da primeira colheita tomou-se uma amostra de dois frutos de cada cultivar em cada subparcela para análise da qualidade avaliada pelo teor de sólidos solúveis (SS).

A análise do teor de sólidos solúveis foi determinada pelo refratômetro digital, a partir de uma fatia do fruto cortado longitudinalmente e colocada num espremedor de frutas manual para obtenção do suco, que foi usado no refratômetro para realização da leitura de SS, expresso em percentagem.

Os dados foram interpretados por meio de análises de variância e da regressão, sendo as médias do fator nitrogênio comparadas através do teste Tukey a 0,05 de probabilidade e os efeitos do fator salinidade avaliados por análise de regressão. Utilizaram-se, para tal, o software SISVAR, versão 5.0 (Ferreira, 2003) e o SAEG versão 9.0 (Ribeiro Júnior et al., 2001).

Para análise dos componentes de produção e qualidade dos frutos as três cultivares (Quetzali, Shadow e Leopard) foram analisadas isoladamente. Estudou-se o efeito das cinco CEa e das três doses de nitrogênio em subparcela $5 \times 3$ para essas características, com quatro repetições.

\section{Resultados E Discussão}

A produção de melancia e seus componentes foram afetados apenas pela $\mathrm{CE}$ da água de irrigação, de maneira isolada (Tabela 1).

Tabela 1. Resumo da ANOVA e valores médios de produtividade, número de frutos por planta e peso médio de frutos comerciais e totais (PRODC, PRODT, NFC, NFT, PMC e PMT) de melancia cultivar Shadow, em função da condutividade elétrica (CE) da água de irrigação e doses de nitrogênio $(N)$ aplicados via fertirrigação

\begin{tabular}{|c|c|c|c|c|c|c|c|}
\hline \multirow{2}{*}{ F.V. } & \multirow{2}{*}{ GL } & \multicolumn{6}{|c|}{ Estatística F } \\
\hline & & PRODC & PRODT & NFC & NFT & PMC & PMT \\
\hline $3 \mathrm{~L}$ & 3 & $5,62^{*}$ & $3,38^{*}$ & $5,42^{* *}$ & $2,35^{\text {ns }}$ & $0,80^{\text {ns }}$ & $2,09^{\text {ns }}$ \\
\hline $\mathrm{CEa}$ & 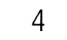 & $2,41^{\mathrm{ns}}$ & $4,78^{* *}$ & $1,46^{\mathrm{ns}}$ & $3,81 *$ & $1,63^{\text {ns }}$ & $2,22^{\mathrm{ns}}$ \\
\hline Linear & 1 & $6,44^{*}$ & $16,75^{* *}$ & $4,76^{*}$ & $14,86^{* *}$ & $0,62^{\text {ns }}$ & $0,34^{\mathrm{ns}}$ \\
\hline Quadrático & 2 & $0,02^{\text {ns }}$ & $0,26^{\text {ns }}$ & $0,094^{\mathrm{ns}}$ & $0,33^{\text {ns }}$ & $0,46^{\text {ns }}$ & $0,10^{\text {ns }}$ \\
\hline Erro (A) & 12 & --- & --- & --. & --- & --. & -- \\
\hline 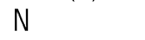 & 2 & $0,74^{\mathrm{ns}}$ & $2,23^{\text {ns }}$ & $1,12^{n}$ & $2,57^{\mathrm{ns}}$ & $0,15^{\text {ns }}$ & $0,73^{\text {ns }}$ \\
\hline $\mathrm{N} \times \mathrm{CEa}$ & - & $0,45^{\text {ns }}$ & $0,97^{\mathrm{ns}}$ & $0,62^{\text {ns }}$ & $1,30^{\text {ns }}$ & $0,49^{\text {ns }}$ & $1,57^{\mathrm{ns}}$ \\
\hline Erro (B) & 30 & \multicolumn{6}{|c|}{ Médias } \\
\hline \multicolumn{2}{|c|}{ Dose de nitrogênio } & \multicolumn{4}{|c|}{$\mathrm{Mg} \mathrm{ha}^{-1}$} & \multicolumn{2}{|c|}{ ing } \\
\hline \multicolumn{2}{|c|}{ N1 $\left(48 \mathrm{~kg} \mathrm{ha}^{-1}\right)$} & $7,49 \mathrm{~A}$ & $44,68 \mathrm{~A}$ & $1,41 \mathrm{~A}$ & $1,80 \mathrm{~A}$ & $4,94 \mathrm{~A}$ & 5,26 \\
\hline \multicolumn{2}{|c|}{ N2 (96 kg ha-1) } & $39,44 \mathrm{~A}$ & $46,71 \mathrm{~A}$ & $1,46 \mathrm{~A}$ & $1,83 \mathrm{~A}$ & $5,07 \mathrm{~A}$ & $5,35 \mathrm{~A}$ \\
\hline \multicolumn{2}{|c|}{ N3 $\left(144 \mathrm{~kg} \mathrm{ha}^{-1}\right)$} & $42,69 \mathrm{~A}$ & $53,48 \mathrm{~A}$ & $1,60 \mathrm{~A}$ & $2,11 \mathrm{~A}$ & $5,08 \mathrm{~A}$ & $5,30 \mathrm{~A}$ \\
\hline \multicolumn{2}{|l|}{ CV1(\%) } & 34,23 & 28,60 & 28,26 & 24,69 & 9,53 & 8,06 \\
\hline \multicolumn{2}{|l|}{ CV2(\%) } & $33,0 /$ & 21,00 & 32,54 & 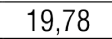 & 68 & 10,65 \\
\hline
\end{tabular}

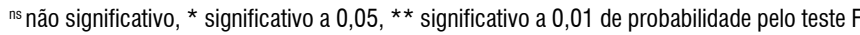
II Médias seguidas de mesma letra na vertical não diferem entre si a 0,05 de probabilidade, pelo teste de Tukey

Verifica-se, ainda, que a salinidade apresentou efeito linear significativo a 0,01 e 0,05 de probabilidade em relação às variáveis PRODT (produtividade total) e PRODC (produtividade comercial) e 0,01 e 0,05 para as variáveis NFT (número de frutos totais) e NFC (número de frutos comerciais) respectivamente (Tabela 1).

Com relação às doses de nitrogênio não houve efeito isolado das mesmas nem da interação nitrogênio x salinidade (Tabela 1). O mesmo não foi observado por Soares (2002) que, estudando o efeito de doses de nitrogênio na melancia, constatou que as diferentes doses influenciaram significativamente sua produtividade.

A partir da equação de regressão estimada na Figura 1A, observa-se que a cultivar Shadow obteve perdas relativas por unidade de salinidade em relação aos valores estimados para a menor salinidade estudada.

Para esta cultivar houve uma redução linear de 2,72 e de 3,38 $\mathrm{Mg} \mathrm{ha}^{-1}$ por incremento unitário na $\mathrm{CE}$ da água de irrigação para 
A.

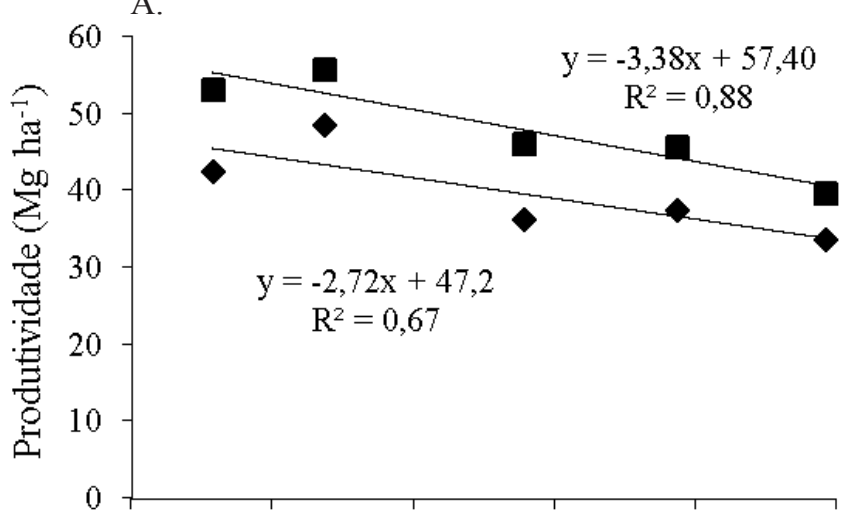

B.

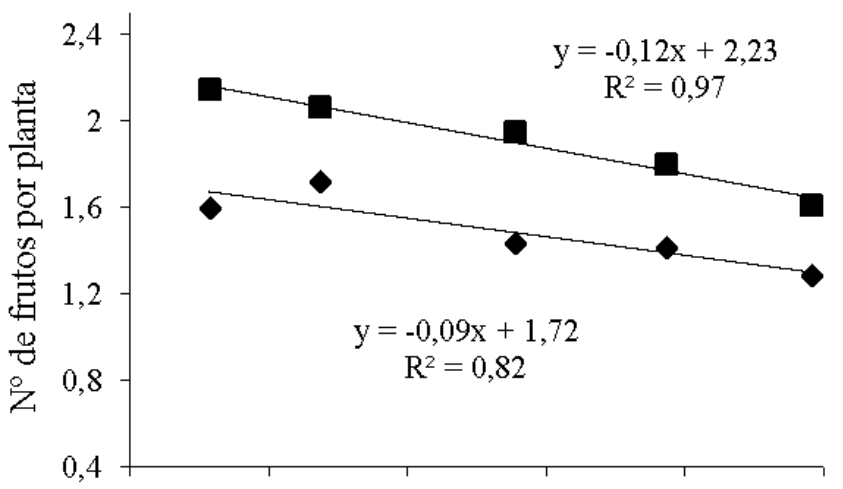

C.

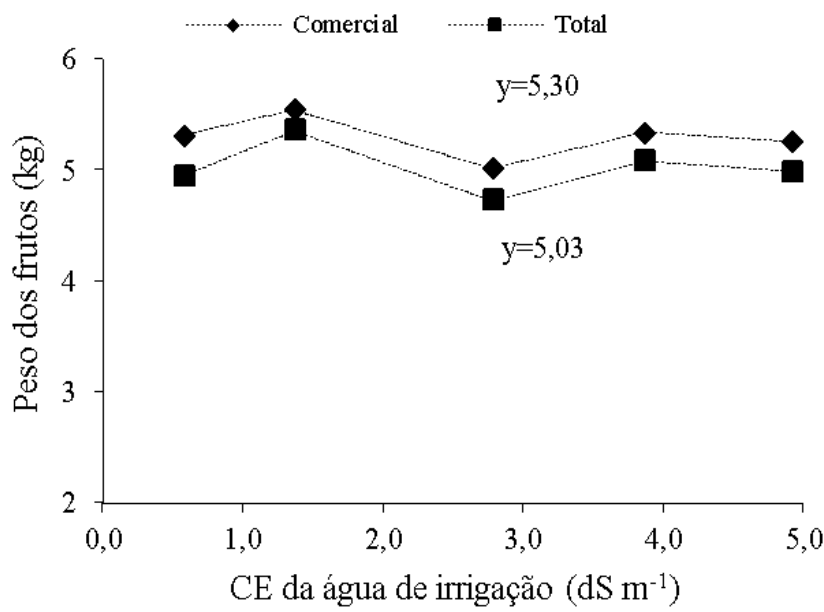

Figura 1. Produtividade $(A)$, número de frutos por planta (B) e peso médio de frutos comercial e total (C) da melancia cultivar Shadow em função da CE da água de irrigação

PRODC e PRODT, respectivamente. A maior produtividade total foi estimada nas plantas irrigadas com água de menor condutividade elétrica $\left(0,57 \mathrm{dS} \mathrm{m}^{-1}\right)$ com uma produtividade de 55,47 $\mathrm{Mg} \mathrm{ha}^{-1}$ (Figura 1A).

De forma semelhante, a maior produtividade comercial foi estimada na menor CEa, com uma produtividade de $45,65 \mathrm{Mg}$ $\mathrm{ha}^{-1}$ correspondente a cerca de $82,3 \%$ da produtividade total. Para ambas as variáveis estudadas a menor produtividade foi observada na maior CEa, com 40,80 e 33,84 $\mathrm{Mg} \mathrm{ha}^{-1}$ resultando, assim, em redução de 26,44 e $25,86 \%$, respectivamente evidenciando, então, que o efeito da condutividade elétrica é mais deletério sobre a produtividade total na maior $\mathrm{CEa}$
(Figura 1A). Carmo (2009) também verificou, estudando a produtividade da melancia irrigada com águas de diferentes níveis de salinidade, que a produtividade da planta foi diminuída linearmente pelos tratamentos salinos.

As equações de regressão do NFC (número de frutos comerciais) e NFT (número de frutos totais) se encontram na Figura 1B. Observa-se que o número de frutos foi reduzido com o aumento da $\mathrm{CE}$ da água de irrigação sendo estimada uma redução de 5,2 e $5,5 \%$, respectivamente, para cada incremento da $\mathrm{CEa}$, ou seja, houve redução de 0,0862 e 0,119 frutos em resposta ao incremento de uma unidade na condutividade elétrica na água de irrigação. O NFC variou de 1,30 a 1,67, respectivamente, para as CEa 4,91 e $0,57 \mathrm{dS} \mathrm{m}^{-1}$ enquanto o NFT variou de 1,65 a 2,17 para as mesmas CEa. Carmo (2009) constatou, estudando o efeito da salinidade no número de frutos por planta de melancia (NF) que os frutos foram reduzidos com o aumento da salinidade da água de irrigação, sendo estimada redução de 0,12 frutos por planta em resposta ao incremento de uma unidade na condutividade elétrica na água de irrigação.

Já a CEa não apresentou efeito significativo sobre o peso médio dos frutos (Figura 1C) obtendo-se o valor médio de 5,3 e 5,03 kg, respectivamente, para peso médio comercial (PMC) e peso médio total (PMT).

As perdas relativas de rendimento comercial e total da cultivar Shadow por incremento unitário da salinidade da água de irrigação foram de 6,0;6,1;5,2 e 5,5\% para PRODC, PRODT, NFC e NFT, respectivamente; assim, as perdas de rendimento são devidas à redução na quantidade de frutos afetada pela salinidade da água de irrigação.

As variáveis rendimento e componentes de produção da cultivar Quetzali foram influenciados linearmente pela $\mathrm{CE}$ da água de irrigação sobre a produtividade comercial e total (PRODC e PRODT) e peso médio de frutos comerciais e totais (PMC e PMT) a nível de 0,01 de probabilidade enquanto as doses de nitrogênio estudados não afetaram significativamente nenhuma das variáveis analisadas (Tabela 2).

A produtividade da melancia Quetzali (Figura 2A) foi reduzida linearmente em resposta ao aumento da condutividade elétrica da água de irrigação. Esta redução foi cerca de 2,29 (PRODC) e 1,99 (PRODT) Mg ha ${ }^{-1}$ por aumento unitário da $\mathrm{CEa}$, estimando a máxima produtividade para as plantas irrigadas com água de menor CEa $\left(0,57 \mathrm{dS} \mathrm{m}^{-1}\right)$ obtendo-se 24,5 e $29,6 \mathrm{Mg} \mathrm{ha}^{-1}$, para uma população de 5000 plantas ha $^{-1}$. O incremento da condutividade elétrica provocou redução total em cerca de 9,93 (PRODC) e 8,63 (PRODT) $\mathrm{Mg} \mathrm{ha}^{-1} \mathrm{o}$ que equivale a 40,6 e $29,1 \%$, respectivamente; desta forma, para a cultivar Quetzali o efeito da condutividade elétrica é mais deletério sobre a produtividade comercial na maior CEa. Carmo (2009) encontrou, trabalhando com a cultivar Quetzali, para a salinidade $0,66 \mathrm{dS} \mathrm{m}^{-1}$ uma produtividade de $80 \mathrm{Mg}$ ha $^{-1}$ enquanto para a salinidade $3,98 \mathrm{dS} \mathrm{m}^{-1}$ a produtividade foi de $48,1 \mathrm{Mg} \mathrm{ha}^{-1}$ para uma população de 10000 plantas $\mathrm{ha}^{-1}$. As perdas quantitativas de produtividade em função da salinidade podem variar com as condições de crescimento, como condições climáticas e de solo, manejo agronômico e de irrigação. 
Tabela 2. Resumo da ANOVA e valores médios de produtividade, número de frutos e peso médio de frutos comerciais e totais (PRODC, PRODT, NFC, NFT, PMC E PMT) de melancia, cultivar Quetzali, em função da CE da água de irrigação e doses de nitrogênio $(\mathrm{N})$ aplicados via fertirrigação

\begin{tabular}{|c|c|c|c|c|c|c|c|}
\hline \multirow{2}{*}{ F.V. } & \multirow{2}{*}{ GL } & \multicolumn{6}{|c|}{ Estatística F } \\
\hline & & PRODC & PRODT & NFC & NFT & PMC & PMT \\
\hline $\mathrm{BL}$ & 3 & $4,95^{* *}$ & $2,5 \mathrm{~ns}$ & $6,35^{\star *}$ & $2,22^{\text {ns }}$ & $0,33^{\mathrm{ns}}$ & $1,12^{\text {ns }}$ \\
\hline $\mathrm{CEa}$ & 4 & 10,95 ns & $7,93 * *$ & $7,68 * *$ & 1,37 ns & $5,74 * *$ & $9,74^{\mathrm{ns}}$ \\
\hline Linear & 1 & $40,98 * *$ & $14,83^{* *}$ & $0,75^{\mathrm{ns}}$ & $2,33 \mathrm{~ns}$ & $17,36^{* *}$ & $35,81 * *$ \\
\hline Quadrático & 2 & $0,49^{\text {ns }}$ & $1,58^{\mathrm{ns}}$ & $0,000093^{\text {ns }}$ & $3,19^{\text {ns }}$ & $4,16^{\text {ns }}$ & $2,50^{\mathrm{ns}}$ \\
\hline $\operatorname{Erro}(A)$ & 12 & -- & --- & --- & --- & -- & -- \\
\hline $\mathrm{N}$ & 2 & $1,58^{\text {ns }}$ & $1,06^{\text {ns }}$ & $3,12^{\text {ns }}$ & $2,13^{\text {ns }}$ & $0,52^{\text {ns }}$ & $0,47^{\mathrm{ns}}$ \\
\hline $\mathrm{N} \times \mathrm{CEa}$ & 8 & $2,19^{\text {ns }}$ & $1,58^{\text {ns }}$ & $1,91^{\mathrm{ns}}$ & $1,48^{\text {ns }}$ & $0,76^{\text {ns }}$ & $1,10^{\text {ns }}$ \\
\hline \multirow[t]{2}{*}{ Erro(B) } & 30 & -- & --- & --- & -- & -- & -- \\
\hline & & \multicolumn{6}{|c|}{ Médias } \\
\hline \multicolumn{2}{|c|}{ Dose de nitrogênio } & \multicolumn{4}{|c|}{$\mathrm{Mg} \mathrm{ha}^{-1}$} & \multicolumn{2}{|c|}{$\mathrm{kg}$} \\
\hline \multirow{3}{*}{\multicolumn{2}{|c|}{$\begin{array}{l}\text { N1 }\left(48 \mathrm{~kg} \mathrm{ha}^{-1}\right) \\
\text { N2 }\left(96 \mathrm{~kg} \mathrm{ha}^{-1}\right) \\
\text { N3 }\left(144 \mathrm{~kg} \mathrm{ha}^{-1}\right)\end{array}$}} & $20,57 \mathrm{~A}$ & $25,50 \mathrm{~A}$ & $1,10 \mathrm{~A}$ & $1,25 \mathrm{~A}$ & $4,49 \mathrm{~A}$ & $4,16 \mathrm{~A}$ \\
\hline & & $18,21 \mathrm{~A}$ & $27,99 \mathrm{~A}$ & $0,76 \mathrm{~A}$ & $1,13 \mathrm{~A}$ & $4,63 \mathrm{~A}$ & $4,22 \mathrm{~A}$ \\
\hline & & $20,08 \mathrm{~A}$ & $26,29 \mathrm{~A}$ & $0,88 \mathrm{~A}$ & $1,30 \mathrm{~A}$ & $4,53 \mathrm{~A}$ & $4,07 \mathrm{~A}$ \\
\hline \multicolumn{2}{|l|}{ CV1(\%) } & 28,47 & 19,30 & 19,94 & 21,23 & 10,38 & 12,43 \\
\hline \multicolumn{2}{|l|}{ CV2(\%) } & 22,68 & 21,01 & 21,91 & 21,75 & 9,31 & 11,64 \\
\hline
\end{tabular}

O número de frutos comercial (NFC) e total (NFT) não foi influenciado pela $\mathrm{CE}$ da água de irrigação apresentando valores médios de 0,85 e 1,23 frutos por planta, respectivamente (Figura 2B). Resultados semelhantes foram encontrados por Uyeda et al. (2005) para a cultura do melão os quais, comparando médias de NFC entre duas cultivares, não verificaram diferença significativa da salinidade em relação a esta variável; as médias de NFC foram de 0,9 frutos por planta.

$\mathrm{O}$ peso médio dos frutos (Figura 2C) foi reduzido com o aumento da condutividade elétrica da água de irrigação sendo estimada redução de 0,160 (comercial) e 0,251 (total) $\mathrm{kg}$ por fruto, em resposta ao incremento de uma unidade na condutividade elétrica na água de irrigação. Para ambos os casos os maiores valores foram observados nas plantas irrigadas com água de menor salinidade $\left(0,57 \mathrm{dS} \mathrm{m}^{-1}\right)$, com 4,9 e $4,7 \mathrm{~kg}$ fruto $^{-1}$ enquanto os menores valores foram estimados para as plantas irrigadas com água de maior salinidade obtendo-se valores de 4,2 e $3,6 \mathrm{~kg}_{\text {fruto }^{-1}}$ e se estimando redução de 14,2 e $23,4 \%$ para PMC e PMT. Carmo (2009) verificou que o peso médio dos frutos foi reduzido com o aumento da salinidade da água de irrigação sendo estimada redução de $0,28 \mathrm{~kg}$ por fruto, em resposta ao incremento de uma unidade na condutividade elétrica da água de irrigação. Os maiores valores foram observados nas plantas irrigadas com água de menor salinidade $\left(0,66 \mathrm{dS} \mathrm{m}^{-1}\right)$ em que o peso médios dos frutos encontrado foi de $4,9 \mathrm{~kg}$ e que, para a água de maior salinidade $\left(3,98 \mathrm{dS} \mathrm{m}^{-1}\right)$ o peso chegou a $3,9 \mathrm{~kg}$.

As perdas relativas por incremento de uma unidade na condutividade elétrica da água de irrigação foram de para 9,$4 ; 6,7 ; 3,3$ e $5,4 \%$ para PRODC, PRODT, PMC, PMT, respectivamente. Assim, o principal responsável pelas perdas de rendimento comercial e total da cultivar Quetzali foi a massa média dos frutos comerciais.
A.

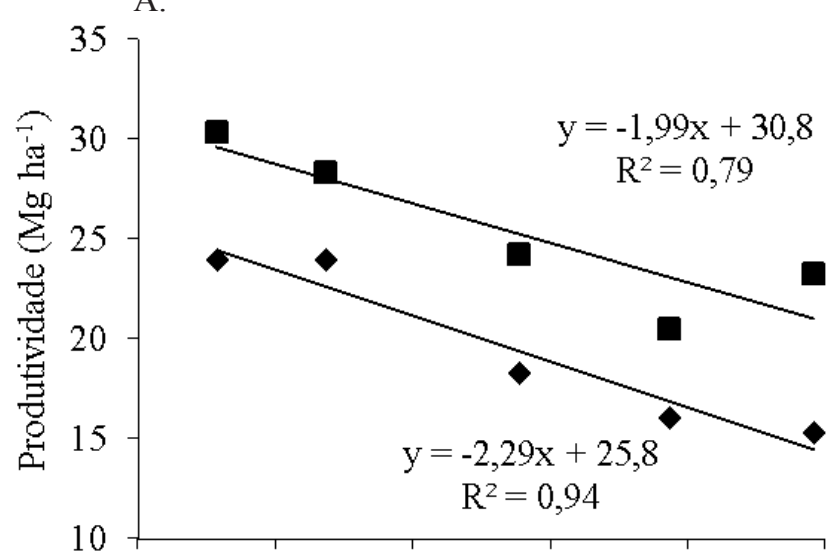

B.

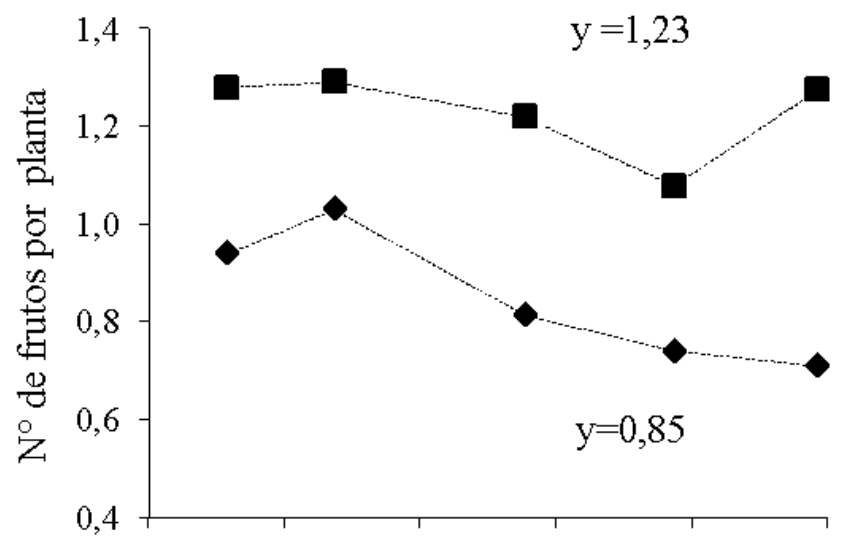

C.

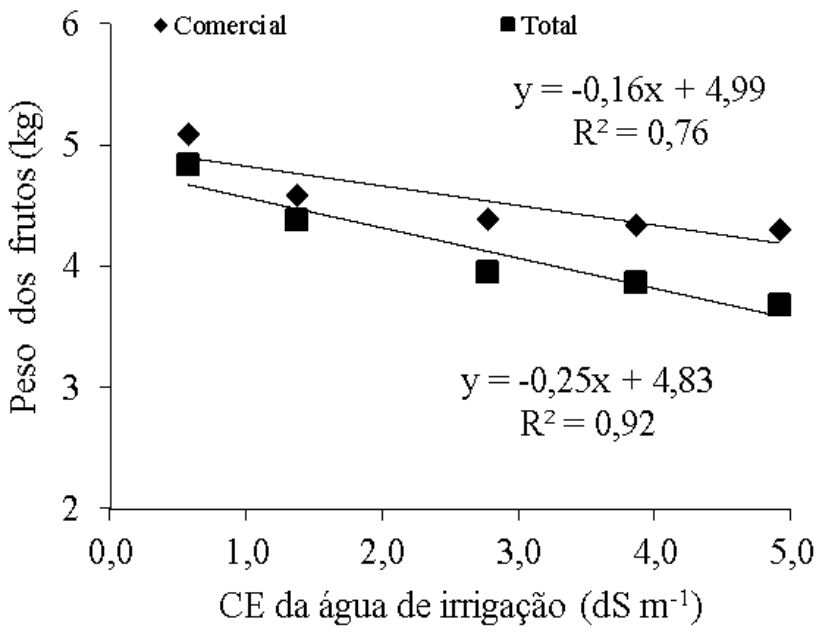

Figura 2. Produtividade (A), número de frutos por planta (B) e peso médio de frutos comerciais e total (C) de melancia cultivar Quetzali, em função da CE da água de irrigação

Aprodução de melancia cultivar Leopard e seus componentes foram afetados pelos fatores isolados (CE da água de irrigação e dose de N) (Tabela 3). Verifica-se que as doses de nitrogênio apresentaram significância para as variáveis de produção das plantas sendo as produtividades comercial e total maiores para a dose de nitrogênio N2. Possivelmente, o aumento da salinidade proporcionado pelo excesso de nitrogênio aplicado (N3) pode ter sido o fator que contribuiu para a produtividade não ter continuado a crescer de N2 para N3. 
Tabela 3. Resumo da ANOVA e valores médios de produtividade, número de frutos e peso médio de frutos comerciais e totais (PRODC, PRODT, NFC, NFT, PMC E PMT) da melancia cultivar Leopard, em função da CE da água de irrigação e doses de nitrogênio $(\mathrm{N})$ aplicadas via fertirrigação

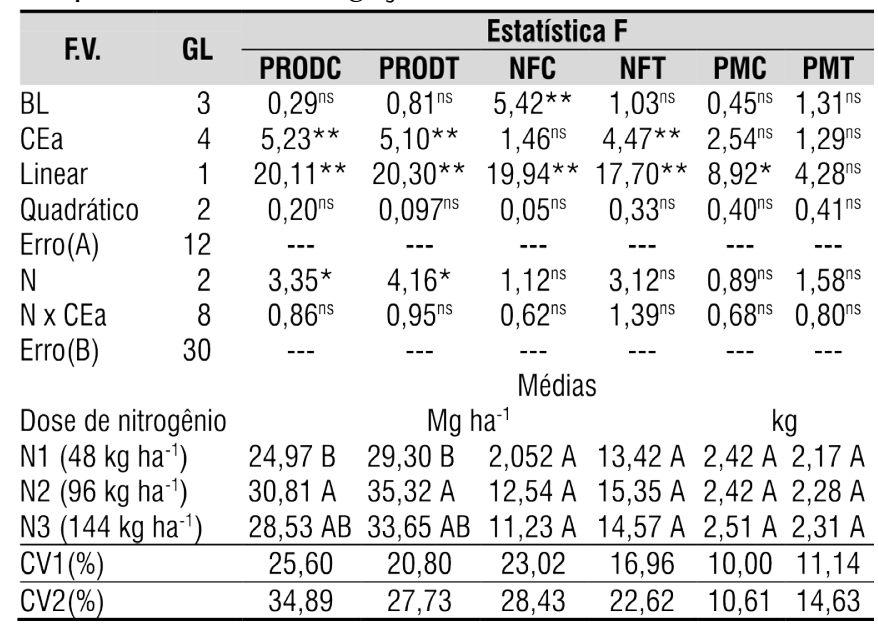

Mousinho et al. (2003) e Araújo et al. (2011) também verificaram efeito significativo do nitrogênio sobre o rendimento da melancia; entretanto, há divergências nas doses de nitrogênio para maximizar as produtividades.

Mousinho et al. (2003) alcançaram, com adubação convencional, produção comercial máxima de 27.477 $\mathrm{kg} \mathrm{ha}^{-1}$ com a dose de $221 \mathrm{~kg} \mathrm{ha}^{-1}$ de N. Araújo et al. (2011) obtiveram um valor máximo de produtividade de $40.428 \mathrm{~kg} \mathrm{ha}^{-1}$ com $144,76 \mathrm{~kg} \mathrm{ha}^{-1}$ de $\mathrm{N}$ indicando que crescentes doses de nitrogênio resultam em aumento de produtividade até um valor máximo, quando há redução na produção a partir deste ponto.

Observa-se também que a CEa apresentou efeito linear significativo para as variáveis produtividade comercial e total (PRODC, PRODT) e número de frutos comerciais e totais (NFC, NFT) a nível de 0,01 de probabilidade e peso médio dos frutos comerciais (PMC) a nível de 0,05 de probabilidade; já o peso médio dos frutos totais (PMT) não sofreu redução proporcional devido ao aumento da CEa (Tabela 3).

Houve uma redução de 3,58 e 3,33 Mg ha ${ }^{-1}$ para as variáveis produtividade comercial (PRODC) e total (PRODT) a cada aumento unitário da CEa (Figura 3A). Em termos percentuais esta redução foi de 43,5 e $36,3 \%$, respectivamente, para a CE da água 4,91 $\mathrm{dS} \mathrm{m}^{-1}$ comparando-se com os valores obtidos pela $\mathrm{CE}$ da água $0,57 \mathrm{dS} \mathrm{m}^{-1}$; o efeito da condutividade elétrica foi, então, mais deletério sobre a produtividade comercial na maior CEa.

As perdas para esta cultivar foram menores que a encontrada por Carmo (2009) para a cultivar Quetzali, já que o autor encontrou uma redução de $9,4{\mathrm{Mg} h{ }^{-1}}$ por aumento unitário da salinidade representando uma redução total em cerca de 39,8\%.

As curvas de respostas dos números de frutos comerciais (NFC) e totais (NFT) em relação à condutividade elétrica da água de irrigação podem ser visualizadas na Figura $3 \mathrm{~B}$ havendo ajuste dos resultados a uma equação linear decrescente. As
A.

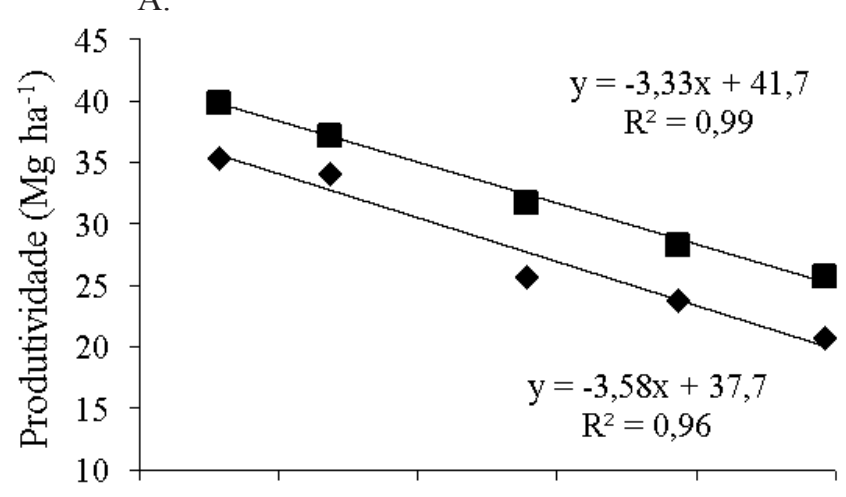

B.

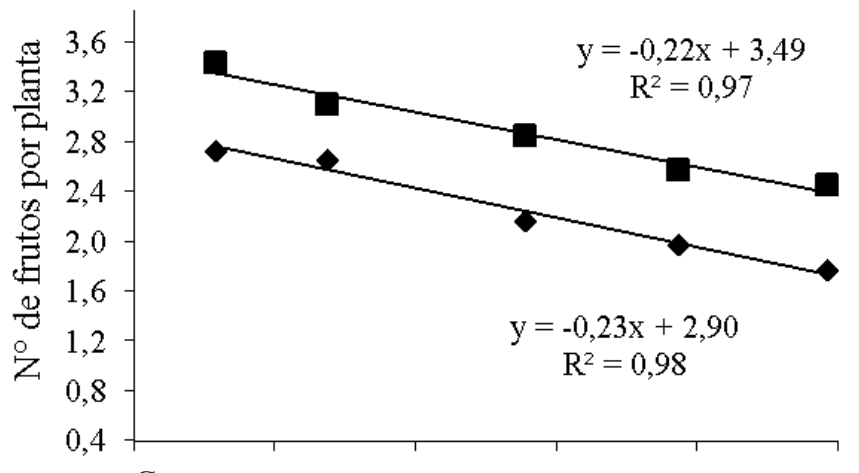

C.

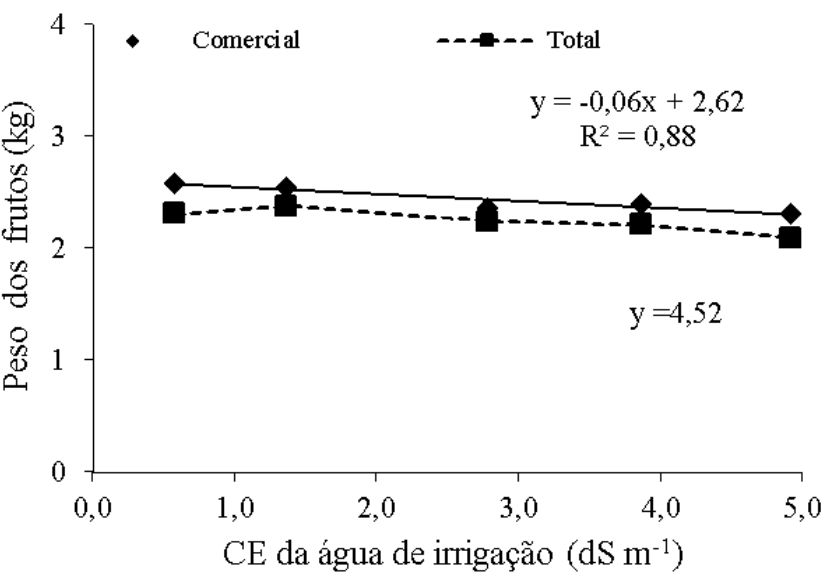

Figura 3. Produtividade (A), número de frutos por planta (B) e peso médio de frutos comerciais e total (C) de melancia, cultivar Leopard, em função da CE da água de irrigação

perdas relativas por unidade de CEa em relação aos valores estimados para a menor CEa estudada, foram de 8,5 e 6,6\% para NFC e NFT, respectivamente.

Em referência à variável massa média dos frutos (Figura 3C) houve uma redução de $0,06 \mathrm{~kg}$ para a massa média dos frutos comerciais (PMC) a cada incremento de $1,0 \mathrm{dS} \mathrm{m}^{-1} \mathrm{da}$ CE da água de irrigação diminuindo em $9,8 \%$ o peso quando comparados seus valores entre a água de irrigação com menor $\mathrm{CE}\left(\mathrm{CE} 1=0,67 \mathrm{dS} \mathrm{m}^{-1}\right)$ com a de maior CE $(\mathrm{CE} 5=4,91 \mathrm{dS}$ $\mathrm{m}^{-1}$ ). Costa et al. (2005) constataram resultados similares trabalhando com diferentes lâminas de irrigação e salinidade da água, para melão tipo amarelo, observando que o rendimento estava relacionado tanto ao peso médio dos frutos como ao número de frutos por planta. 
As perdas relativas foram de $10,8,4,8,5,6,6$ e $2,4 \%$ por incremento unitário na condutividade elétrica da água de irrigação para PRODC, PRODT, NFC, NFT e PMC, respectivamente, ou seja, o número de frutos total e comercial foi a principal variável responsável pela perda de rendimento da produção.

Na Tabela 4 encontra-se um resumo da ANOVA para o teor de sólidos solúveis das cultivares de melancia observando-se que não houve efeito significativo do fator individual $\mathrm{N}$ nem da interação $(\mathrm{CEa} \times \mathrm{N})$ para as três cultivares em relação à qualidade química dos frutos, no que se refere aos teores de sólidos solúveis (SS).

Resultados similares foram encontrados por Andrade Júnior et al. (2006) trabalhando com diferentes doses de nitrogênio na cultura da melancia que verificaram não haver efeito significativo para tal característica porém esses resultados diferem dos de Mousinho et al. (2003) que observaram, em experimento no município de Fortaleza, CE, que a aplicação de diferentes doses de nitrogênio $(0,75,150$ e $300 \mathrm{~kg} \mathrm{ha}^{-1}$ ) influenciou os sólidos solúveis em frutos de melancia reduzindo-os com o aumento da dose e Araújo et al. (2011) que obtiveram um valor máximo de $12,23 \% \mathrm{~kg}$ por fruto, com $162,7 \mathrm{~kg} \mathrm{ha}^{-1}$ de $\mathrm{N}$.

Tabela 4. Resumo da ANOVA para sólidos solúveis $(\%)$ de cultivares de melancia irrigadas com diferentes condutividades elétricas da água de irrigação (CEa) e doses de nitrogênio $(\mathrm{N})$ aplicados em fertirrigação

\begin{tabular}{|c|c|c|c|c|}
\hline \multirow{3}{*}{ F.V. } & \multirow{3}{*}{ G.L. } & \multicolumn{3}{|c|}{ Estatística F } \\
\hline & & \multicolumn{3}{|c|}{ Cultivares } \\
\hline & & Leopard & Quetzali & Shadow \\
\hline $\mathrm{BI}$ & 3 & $4,77^{\star \star}$ & $2,61^{\mathrm{ns}}$ & $0,63^{\text {ns }}$ \\
\hline CEa & 4 & $1,78^{\mathrm{ns}}$ & $1,11^{\text {ns }}$ & $3,13^{\star}$ \\
\hline Linear & & $1,47^{\mathrm{ns}}$ & $0,18^{\text {ns }}$ & $4,92^{\star}$ \\
\hline Quadr. & & $4,72^{\mathrm{ns}}$ & $3,42^{\text {ns }}$ & $4,68^{\star}$ \\
\hline $\operatorname{ERRO}(\mathrm{A})$ & 12 & & & \\
\hline Nitrogênio (N) & 2 & $0,69^{\text {ns }}$ & $0,64^{\text {ns }}$ & $0,37^{\mathrm{ns}}$ \\
\hline$S^{*} N$ & 8 & $0,68^{\text {ns }}$ & $1,37^{\mathrm{ns}}$ & $0,53^{\text {ns }}$ \\
\hline \multirow[t]{3}{*}{ ERRO (B) } & 30 & & & \\
\hline & & \multicolumn{3}{|c|}{ Média geral } \\
\hline & & 10,04 & 10,17 & 9,77 \\
\hline C.V. (A) & & 5,40 & 6,10 & 5,50 \\
\hline C.V. (B) & & 7,10 & 5,10 & 8,00 \\
\hline
\end{tabular}

O efeito da salinidade influenciou o teor de sólidos solúveis dos frutos apenas para a cultivar Shadow sendo o modelo quadrático o de melhor ajuste (Figura 4).

Com o acréscimo nos níveis de salinidade da água de irrigação de 2,77 a $4,91 \mathrm{dS} \mathrm{m}^{-1}$ os valores de SS aumentaram em 3,58 e 5,08\%, respectivamente (Figura 4). O aumento dos níveis de salinidade da água de irrigação reduziu a absorção de água pela planta proporcionando uma concentração maior de SS no fruto. Botía et al. (2005) e Mascarenhas et al. (2010) também verificaram, trabalhando com melão, que os valores de SS aumentam com o acréscimo nos níveis de salinidade da água de irrigação. Para o teor de sólidos solúveis o valor preconizado na literatura como sendo o teor mínimo para obtenção do sabor aceitável em melancia, é de $10^{\circ}$ Brix (Lima Neto et al., 2010). O valor de sólidos solúveis encontrado por Grangeiro \& Cecílio Filho (2004) quando avaliaram o híbrido Tide no estado de São

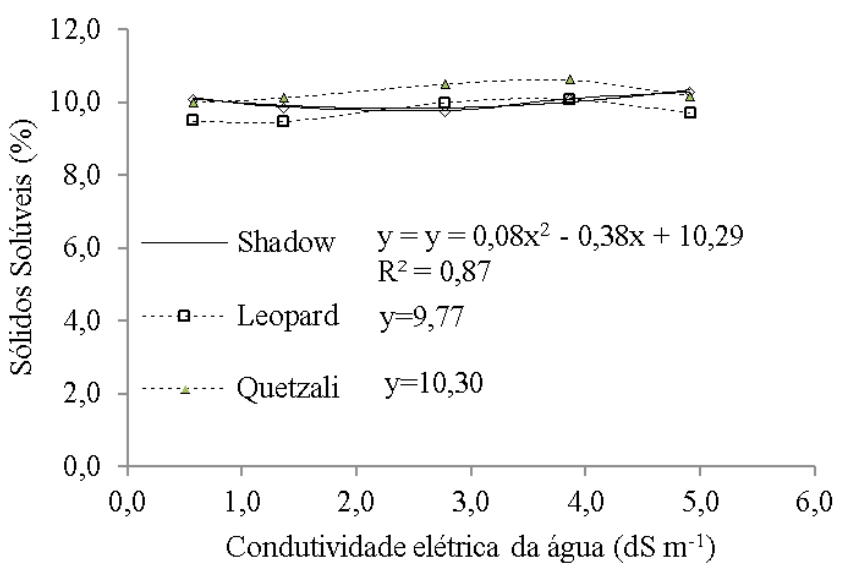

Figura 4. Teores de sólidos solúveis em três cultivares de melancia irrigadas com diferentes níveis salinos da água deirrigação

Paulo, foi superior ao encontrado neste trabalho $(11,2$ a 12,0 ${ }^{\circ}$ Brix). Referida diferença pode ser atribuída às diferenças entre o local de plantio, cultivares, manejo da irrigação e sistema de produção adotado no experimento.

\section{Conclusões}

1. Não houve efeito significativo das doses de nitrogênio na produção das cultivares Shadow e Quetzali enquanto a cultivar Leopard apresentou produtividade comercial e total e número de frutos comerciais maiores para a dose de nitrogênio $96 \mathrm{~kg} \mathrm{ha}^{-1}$.

2. A redução na produção das cultivares Shadow e Leopard em função do aumento da condutividade elétrica da água de irrigação ocorreu em consequência da diminuição do número de frutos.

3. O principal responsável pelas perdas de rendimento comercial e total da cultivar Quetzali foi o peso médio dos frutos.

4. Apenas a cultivar Shadow respondeu ao efeito da condutividade elétrica da água de irrigação sobre o teor de sólidos solúveis dos frutos.

5. A cultivar Shadow foi a mais tolerante em relação ao efeito da condutividade elétrica da água de irrigação visto que apresentou menor perda relativa de produção comercial.

\section{Agradecimentos}

Os autores agradecem ao projeto FRUTERN - Convênio FINEP/ESAM/FGD, pela infraestrutura de campo e ao CTARN - Convênio FINEP/FAPERN/UFERSA/ EMPARN/UFRN, pela infraestrutura laboratorial; ao $\mathrm{CNPq}$, que disponibilizou os recursos necessários à realização deste projeto e à CAPES, pela concessão da bolsa de estudo.

\section{Literatura Citada}

Allen, R. G.; Pereira, L. S.; Raes, D.; Smith. Evapotrasnpriation del cultivo: Guias para a la determinacion de los requeriments de água de los cultivos. Roma: FAO, 2006. 298p. 
Andrade Júnior, A. S.; Dias, N. da S.; Figueiredo Júnior, L. G. M. F.; Ribeiro, V. Q.; Sampaio, D. B. Produção e qualidade de frutos de melancia à aplicação de nitrogênio via fertirrigação. Revista Brasileira de Engenharia Agrícola e Ambiental, v.10, p.836-841, 2006.

Andrade Júnior, A. S.; Dias, N. S.; Figueredo Junior, L. G. M.; Daniel, R.; Ribeiro, V. Q. Doses de potássio via fertirrigação na produção e qualidade de frutos de melancia em Parnaíba, PI. Irriga, v.10, p.205-214, 2005.

Araújo, W. F.; Barros, M. M.; Medeiros, R. D.; Chagas, E. A.; Neves, L. T. B. C. Crescimento e produção de melancia submetida a doses de nitrogênio. Revista Caatinga, v.24, p.80-85, 2011.

Botía, P.; Navarro, J. M.; Cerdá, A.; Martínez, V. Yield and fruit quality of two melon cultivars irrigated with saline water at different stages of development. European Journal of Agronomy, v.23, p.243-253, 2005.

Carmo, G. A. do. Crescimento, nutrição e produção de cucurbitáceas cultivadas sob diferentes níveis de salinidade da água de irrigação e doses de adubação nitrogenada. Mossoró: UFERSA, 2009. 182p. Tese Doutorado

Costa, A. R. F. C.; Medeiros, J. F. de; Porto Filho, F. Q.; Silva.; J. S.; Freitas, D. C.; Costa, F. G. B. Produção de cultivares de melancia submetidas a níveis de salinidade da água de irrigação e doses de nitrogênio. Revista Brasileira de Agricultura Irrigada, v.4, p.242-248, 2010.

Costa, F. A.; Medeiros, J. F. de; Negreiros, M. Z. Rendimento de melão cantaloupe em diferentes coberturas de solo e lâminas de irrigação. Revista Caatinga, v.15, p.49-55, 2005.

EMPRAPA - Empresa Brasileira de Pesquisa Agropecuária. Sistema brasileiro de classificação de solo. Brasília: EMBRAPA, 1999. 412p.

Ferreira, D. F. SISVAR, versão 5.0 (Build 67), DEX/FLA, 2003.

Ferreira, M. A. J. F.; Queiroz, M. A.; Braz, L. T.; Vencovsky, R. Correlações genotípicas, fenotípicas e de ambiente entre dez caracteres de melancia e suas implicações para o melhoramento genético. Horticultura Brasileira, v.21, p.438-442, 2003.

Figueirêdo, V. B. Evapotranspiração, crescimento e produção da melancia e melão irrigados com águas de diferentes salinidades. Botucatu: UNESP, 2008. 104p. Tese Doutorado

Grangeiro, L. C.; Cecílio Filho, A. B. Qualidade de frutos de melancia em função de fontes e doses de potássio. Horticultura Brasileira, v.22, p.647-650, 2004.
Kafkafi, U. Plant nutrition under saline conditions. In: Shainber, G. I.; Shalhevet, K. Soil salinity under irrigation. Berlin: Springer-Verlag, 1984. p.319-338.

Lima Neto, I. S.; Guimarães, I. P.; Batista, P. F.; Aroucha, E. M. M.; Queiroz.; M. A. Qualidade de frutos de diferentes variedades de melancia provenientes de Mossoró-RN. Revista Caatinga, v.23, p.14-20, 2010.

Mascarenhas, F. R.; Medeiros, D. C.; Medeiros, J. F. de; Dias, P. M. S.; Souza.; M. S. M. Qualidade de melão gália cultivado sob diferentes níveis de salinidade. Revista Verde de Agroecologia e Desenvolvimento Sustentável, v.5, p.171-181, 2010.

Medeiros, J. F. de; Lisboa, R. de A.; Oliveira, M.; Silva Júnior, M. J. da; Alves, L. P. Caracterização das águas subterrâneas usadas para irrigação na área produtora de melão da Chapada do Apodi. Revista Brasileira de Engenharia Agrícola e Ambiental, v.7, p.469-472, 2003.

Miguel, A. C. A.; Dias, J. R. P. S.; Spoto, M. H. F. Efeito do cloreto de cálcio na qualidade de melancias minimamente processadas. Horticultura Brasileira, v.25, p. 442-446, 2007.

Mousinho, F. E. P.; Costa, R. N. T.; Souza, F.; Gomes Filho, R. R. Função de resposta da melancia à aplicação de água e nitrogênio para as condições edafoclimáticas de Fortaleza, CE. Irriga, v.8, p.264-272, 2003.

Queiroga, R. C. F. de; Andrade Neto, R. de C.; Nunes, G. H. de S.; Medeiros, J. F. de; Araújo, W. de B. M. de. Germinação e crescimento inicial de híbridos de meloeiro em função da salinidade. Horticultura Brasileira, v.24, p.315-319, 2006.

Ribeiro Júnior, J. I. Análises estatísticas no SAEG. Viçosa, MG: UFV, 2001.301p.

Torres, S. B. Germinação e desenvolvimento de plântula de melancia em função da salinidade. Revista Brasileira de Sementes, v.29, p.77-82, 2007.

Uyeda, C. A.; Gurgel, M. T.; Gheyi, H. R.; Oliveira; F. H. T. de; Fernandes, P. D. Produtividade de duas cultivares de meloeiro irrigadas com água de alta e baixa salinidade. Revista Brasileira de Engenharia Agrícola Ambiental, v.9, p.318-321, 2005.

Soares, J. I.; Costa, R. N. T.; Silva, L. A. C.; Gondim, R. S. Função de resposta da melancia aos níveis de água e adubação nitrogenada, no Vale do Curu, CE. Revista Brasileira de Engenharia Agrícola e Ambiental, v.9, p.219-224, 2002.

Viana, S. B. A.; Fernandes, P. D.; Gheyi, H. R.; Soares, F. A. L.; Carneiro, P. T. Índices morfofisiológicos e de produção de alface sob estresse salino. Revista Brasileira de Engenharia Agrícola e Ambiental, v.8, p.23-30, 2004. 